Trauma Berufskrankh $2005 \cdot 7[$ Suppl 1]:S134-S138 DOI 10.1007/s10039-004-0943-0

Online publiziert: 5. November 2004

๑) Springer Medizin Verlag 2004

J. Wodtke · D. Klüber · L. Frommelt · J. F. Löhr · ENDO-Klinik, Hamburg

\title{
Die infizierte Gelenkendoprothese
}

on zu verzeichnen ohne kurzfristige Aussichten auf wesentliche Fortschritte. Eine gute Erfolgsrate liegt bei ca. $85 \%$ (• Tabelle 2).

\section{Wirtschaftliche Aspekte}

Versorgungsengpass. War es bislang in erster Linie ein schwerer Leidensweg für die Patienten und eine Herausforderung an den Chirurgen, so wird jetzt die Infektionsbehandlung zunehmend zu einem wirtschaftlichen Problem unter Einbeziehung des einzelnen Patienten. Maßgeblich hierfür ist, dass die enormen zusätzlichen Therapiekosten im DRG-System nicht abgebildet und bei der Vergütung durch die Kostenträger infolgedessen negiert werden. Diese Praxis limitiert die möglichen Behandlungszahlen bei großen Kliniken deutlich, und kleine Häuser werden die Verluste, die bei jeder Behandlung gegenüber einer „aseptischen“ Revision rechne- risch anfallen, nicht mehr tragen können. Der so verursachte Versorgungsengpass lässt eine zunehmende Wartezeit für Patienten mit akut zu versorgenden Krankheitsbildern entstehen, die sich deutlich verschlimmernd auswirkt und sogar zu Todesfällen führt, die aus verzögerter Behandlung resultieren.

Inadäquate Behandlung. Inadäquate $\mathrm{Be}-$ handlungsversuche wie Fistelexzisionen, Spülungen, Ketteneinlagen, Polyäthylenkomponentenwechsel, Vakuumversiegelungen etc. als Überbrückungstherapien bleiben weiterhin bestehen und verschlechtern die Ausgangssituation für den letztlich doch erforderlichen Sanierungseingriff.

Diese Kleinsteingriffe werden im DRGSystem sehr gut vergütet (• Tabelle 3$)$.

Ein wirtschaftlicher Aspekt soll dieser Handlungsweise nicht unterstellt werden, zumal es sich um eingefahrene Therapieverist hier seit Jahren eine deutliche Stagnati-

\begin{tabular}{|llc}
\hline \multicolumn{3}{|l}{ Tabelle 1 } \\
$\begin{array}{l}\text { Übersicht Infektraten bei } \\
\text { Primär-TEP }\end{array}$ \\
\hline Gluck & 1891 & $100 \%$ \\
\hline Charnley & 1964 & $10 \%$ \\
\hline Buchholz & 1969 & $3 \%$ \\
\hline $\begin{array}{l}\text { Crockarell, } \\
\text { Mayo Clinic }\end{array}$ & 1998 & $1,4 \%$ \\
\hline ENDO-Klinik & 1999,2001, & $<1 \%$ \\
\hline Mohr & 2002,2003 & \\
\hline
\end{tabular}

Tabelle 2

Erfolgsraten aus der Literatur für Hüft-TEP-Wechsel bei Infektion

\begin{tabular}{|lccll}
\hline Autoren & Jahr & Anzahl Patienten & Einzeitig & Zweizeitig \\
Carlson et al. & 1978 & 77 & $78 \%$ & \\
\hline Buchholz et al. & 1981 & 583 & $77 \%$ & \\
\hline James et al. & 1982 & 1063 & $73 \%$ & \\
\hline Fitzgerald/Jones & 1985 & 131 & & $88 \%$ \\
\hline Langelais et al. & 1986 & $177 / 222$ & $88 \%$ & $85 \%$ \\
\hline Coyler/Kapello & 1994 & 37 & & $84 \%$ \\
\hline Raut et al. & 1995 & 183 & $84 \%$ & \\
\hline Steinbrink/Frommelt & 1995 & 163 & $88 \%$ & \\
\hline Wodtke/Luck/Löhr & 2003 & 105 & $88 \%$ & \\
\hline
\end{tabular}


suche mit langer Tradition handelt. Für die Kostenträger ist diese Handlungsweise grob unwirtschaftlich, da viele stationäre Aufenthalte abgerechnet werden und die erforderliche kurative Therapie verzögert und in der Wirksamkeit beeinträchtigt wird. Die Gesamtkosten liegen dadurch weit über einer planvoll und zeitgerecht durchgeführten Behandlung. Auch dieses Argument bleibt bei den Kostenträgern bislang ohne Resonanz. Gleichbehandlung bei dem Erstattungsbetrag eines Prothesenwechsels bei infizierter und aseptischer Situation, wie z. B. beim Kniegelenk ist absurd, da die adäquate Infektbehandlung erhebliche Zusatzkosten verursacht. Hierzu 2 treffende Zitate:

\section{“... die Preise werden bundes- weit vorgegeben für eine bestimmte Leistung, und das Krankenhaus muss seine Kosten entsprechend anpassen oder kann die Behandlung nicht mehr anbieten" [6].}

Die Umsetzung dieser Erkenntnis wäre für die Patientenversorgung fatal und kann seitens des Gesetzgebers so nicht gewollt sein. Auch in den USA besteht seit langem die gleiche Problematik, sodass Sculco [14] sich schon 1993 entsprechend geäußert hat:

\section{7) “... rapid, aggressive and definitive treatment must be rendered to the patient. ... Reimbursement to hospitals must more realistically reflect the magnitude of resources consumed by these patients."}

Kostenvergleich. Ein direkter Kostenvergleich ist mit vielen diskutierbaren Variablen verbunden, aber Zusatzkosten der Wechseleingriffe bei infizierten Gelenken lassen sich leicht beispielhaft aufzählen. Längere Operationszeiten und erhöhter Personalaufwand sind deutlich kostenträchtig. Ein besonders plausibles Beispiel zur Verdeutlichung der Mehrkosten ist der Antibiotikaverbrauch. Der einzeitige Prothesenwechsel - seit über 30 Jahren anerkanntes Therapieverfahren - erfordert eine topische und systemische Antibiotikagabe. Auch der mehrzeitige Revisionseingriff

Tabelle 3

Kleinsteingriffe ${ }^{a}$ : Erlöse nach DRG Baserate 2004

\begin{tabular}{|ll}
\hline Wechsel TEP „,aseptisch“ ohne Nebendiagnosen & 7900,- EUR \\
\hline Wechsel TEP „infiziert“ ohne Nebendiagnosen & $9500,-$ EUR \\
\hline Wundrevision bei infizierter TEP ohne Nebendiagnosen & $4777,-$ EUR \\
& \\
&
\end{tabular}

Tabelle 4

Antibiotikakosten ${ }^{\mathrm{a}}$ in EUR bei Verwendung von $160 \mathrm{~g}$ Zement/Patient/

Keimsituation

\begin{tabular}{|lccc}
\hline & Zementzusatz & $\begin{array}{l}\text { Systemische } \\
\text { Antibiotikatherapie }\end{array}$ & Gesamtkosten \\
\hline Staphylococcus sp., empfindlich & 748,00 & 440,22 & 1188,22 \\
\hline Staphylococcus sp., resistent & 1801,88 & 1238,13 & 3040,01 \\
\hline S. aureus (MRSA) & 1801,88 & 3855,92 & 5657,80 \\
\hline Streptococcus sp. & 748,00 & 545,21 & 1293,21 \\
\hline Propionibacterium sp. & 748,00 & 350,20 & 1098,20 \\
\hline Enterococcus sp. & 1488,40 & 744,74 & 2233,14 \\
\hline Pseudomonas aeruginosa & 2203,04 & 3333,43 & 5536,47 \\
\hline Unbekannter Keim & 1467,68 & 507,18 & 1974,86 \\
\hline a nach:C. Hendrich und L. Frommelt, Würzburg 2003 & & \\
\hline
\end{tabular}

Tabelle 5

Erlöse beim Hüftendoprothesenwechsel, abzüglich Antibiotikakosten

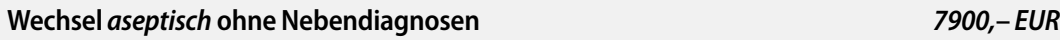
(DRG Baserate 2004)

\begin{tabular}{|c|c|c|}
\hline Wechsel infiziert wie oben, einzeitig & 9500,-EUR & \\
\hline Abzüglich Antibiotikakosten, z. B. für Staph. sp., resistent & $-3040,-$ EUR & $6460,-E U R$ \\
\hline $\begin{array}{l}\text { Wechsel infiziert wie oben, zweizeitig, ein stationärer } \\
\text { Aufenthalt }\end{array}$ & 10.520,-EUR & \\
\hline $\begin{array}{l}\text { Abzüglich doppelter Antibiotikakosten, } \\
\text { z. B. für Staph. sp., resistent }\end{array}$ & $-6080,-$ EUR & $4440,-E U R$ \\
\hline $\begin{array}{l}\text { Wechsel infiziert wie oben, zweizeitig, } \\
2 \text { stationäre Aufenthalte }\end{array}$ & 14.544,-EUR & \\
\hline $\begin{array}{l}\text { Abzüglich doppelter Antibiotikakosten, } \\
\text { z. B. für Staph. sp., resistent }\end{array}$ & $-6080,-$ EUR & $8464,-E U R$ \\
\hline
\end{tabular}

wird regelhaft mit topischer und systemischer Antibiotikatherapie kombiniert, sodass bei genauer Auslegung dieses Verfahrens sogar die doppelten Kosten anfallen. Hendrich und Frommelt [11] haben die Preise für die Antibiotika bei der Verwendung von $160 \mathrm{~g}$ Knochenzement und die adjuvante systemische Therapie für unterschiedliche Keime zusammengestellt (• Tabelle 4).

Vergleicht man nun die Erlöse der gängigen chirurgischen Therapieverfahren mit denen der aseptischen Revision und bringt nur die Kosten für die erforderlichen Antibiotika in Abzug, zeigen sich deutliche Verluste in nur einem beispielhaften Punkt (• Tabelle 5).

Lediglich ein zweizeitiger Wechsel mit 2 stationären Aufenthalten liegt bei Anrechnung des Antibiotikaverbrauchs in der Erstattung über dem aseptischen Wechsel. Hier muss natürlich auch die längere stationäre Versorgung einbezogen werden. Bei der üblichen Verfahrenswei- 
Trauma Berufskrankh 2005 · 7[Suppl 1]:S134-S138

DOI 10.1007/s10039-004-0943-0

(C) Springer Medizin Verlag 2004

J. Wodtke · D. Klüber · L. Frommelt · J. F. Löhr

\section{Die infizierte Gelenkendoprothese}

\section{Zusammenfassung}

Als schwerste Komplikation der Gelenkendoprothetik bleibt die periprothetische Infektion eine Herausforderung - heutzutage nicht nur für den Chirurgen, sondern als finanzielles Problem für die Versorger und Kostenträger. Insbesondere die Patienten leiden unter dem sich abzeichnenden Versorgungsengpass. Bei regelhafter Anwendung der einzeitigen Wechselstrategie als Therapie der Wahl in dafür geeigneten und zu bildenden Zentren ließen sich alle Vortei-

\section{Periprosthetic joint infection}

\begin{abstract}
Periprosthetic infection remains the most severe complication in joint arthroplasty. Always a hardship for the patient and still a challenge for the surgeon, it is a rapidly growing burden for the economy. Unrealistically low reimbursement to the individual clinic by the healthcare system leads to a remarkable shortage in acute treatment possibilities. The options for treatment according to the indications are clear. Following the theory of Costerton and Gristina a complete exchange of the foreign body is mandatory accompanied by radical débridement. Antibiotic augmentation of the bone cement
\end{abstract}

le dieses Verfahrens ausschöpfen. Bei deutlich abgesenktem Kostenniveau können bessere Ergebnisse erreicht werden. Primär ist jedoch eine realistische Anhebung der Vergütungsbeträge auf das tatsächliche Kostenniveau Voraussetzung.

\section{Schlüsselwörter}

Gelenkendoprothetik · Infektion . Kosten der Infekttherapie · Komplikation . Versorgungsengpass

and adjuvant systemic antibiotic treatment allow a one-stage exchange with good functional results that satisfy the patients. Prerequisites are a well-experienced surgical team in close cooperation with an equally specialised microbiologist and his laboratory in a centre with sufficient resources provided by the healthcare system.

\section{Keywords}

Joint arthroplasty - Periprosthetic infection . Cost of treatment of infection . Complication · Shortage in treatment possibilities se, z. B. dem einzeitigen Wechsel, entsteht ein rechnerischer Verlust von 1440,- EUR. Weitere Kosten wie oben erwähnt bleiben hier erst einmal unberücksichtigt.

Noch absurder ist die Situation beim Kniegelenkwechsel wegen Infektion, da hier die Erstattung gleich wie beim aseptischen Eingriff ist. Die Wundrevision wird jedoch überproportional hoch vergütet (- Tabelle 6).

Bringt man nun noch die rechnerischen Kosten für die verlängerte Op.-Zeit in Ansatz (mit ca. 30,-EUR/min = 1800,$\mathrm{EUR} / \mathrm{h}$ ), ist klar, dass kurzfristig eine adäquate Anpassung erfolgen muss.

Aus der Sicht unserer Spezialklinik hat sich die Situation des Versorgungsengpasses für Patienten mit periprothetischen Infektionen im Laufe der letzten 2-3 Jahre deutlich verschärft. Eine Lösung dieses Problems wurde seitens der Kostenträger bzw. des Gesetzgebers bislang nicht in Aussicht gestellt.

\section{Medizinische Aspekte}

Die medizinisch-chirurgische Therapie der periprothetischen Infektion ist klar definiert mit verschiedenen Optionen bei entsprechenden Indikationen (• Tabelle 7).

Der Prothesenerhalt gelingt bei sofortiger Intervention früh postoperativ zu einem vertretbaren Prozentsatz $[5,15]$. Erforderlich ist ein radikales Débridement und die Anlage einer Spül-Saug-Drainage in Verbindung mit einer systemischen Antibiose. Nicht alle Keime lassen sich gleich erfolgreich therapieren. Die besten Chancen bestehen bei Streptokokken, gefolgt von Staphylokokken.

Die allein antibiotische Suppressionstherapie [16] ist nur in den genannten Ausnahmesituationen ersatzweise zu versuchen, wenn eine Operation nicht durchgeführt werden kann oder soll. Hierunter kann es durchaus zu langanhaltenden beschwerdearmen Intervallen kommen. Bei Absetzen der Therapie ist mit Wiedereinsetzen der Symptome zu rechnen. Teilweise lässt sich jedoch kein Einfluss auf die Infektion nehmen.

Die Resektion einer prothesenversorgten Extremität als Ultima ratio in lebensbedrohlicher Situation ist der Einzelfall und nicht als Therapie zu propagieren. 


\begin{tabular}{|l|}
\hline Tabelle 6 \\
\hline $\begin{array}{l}\text { Erstattungen bei der } \\
\text { Kniegelenksrevision }\end{array}$ \\
\hline $\begin{array}{l}\text { Wechsel Knieprothese } \\
\text { aseptisch }\end{array}$ \\
\hline $\begin{array}{l}\text { Wechsel Knieprothese } \\
\text { infiziert }\end{array}$ \\
\hline $\begin{array}{l}\text { Wundrevision Knie } \\
\text { (z. B. Spülung, Ketteneinlage, -EUR } \\
\text { etc.) }\end{array}$ \\
\hline
\end{tabular}

Tabelle 7

Therapeutische Optionen bei periprothetischer Infektion Indikationen

\begin{tabular}{|ll}
$\begin{array}{l}\text { Prothesen- } \\
\text { erhalt }\end{array}$ & $\begin{array}{l}\text { Bei Akutinfekt bis } 2 \text { Wochen } \\
\text { postoperativ }\end{array}$ \\
\hline $\begin{array}{l}\text { Prothesen- } \\
\text { wechsel }\end{array}$ & $\begin{array}{l}\text { Einzeitig:,,golden standard“ } \\
\text { Zweizeitig: bei unsicheren } \\
\text { Keimsituationen } \\
\text { - Bei speziellen Resistenz- } \\
\text { lagen } \\
\text { - Bei Allergien auf erforder- } \\
\text { liche Antibiotika } \\
\text { - Bei extremer Ausdehnung } \\
\text { der Infektion }\end{array}$ \\
\hline $\begin{array}{l}\text { Suppres- } \\
\text { sion }\end{array}$ & $\begin{array}{l}\text { Bei Patienten, die nicht } \\
\text { operationsfähig sind } \\
\text { Bei Patienten, die nicht } \\
\text { operationswillig sind }\end{array}$ \\
\hline Resektion & $\begin{array}{l}\text { Bei Versagen aller vorheri- } \\
\text { gen Therapieversuche im } \\
\text { Notfall }\end{array}$ \\
\hline
\end{tabular}

\section{Wechselkonzept - Therapie der Wahl}

Der einzeitige Prothesenwechsel - nach unserer Auffassung die Therapie der Wahl bei periprothetischen Infekten - soll im Folgenden kurz skizziert werden:

Pathogenese. Die Pathogenese der periprothetischen Infektion unterscheidet sich von der anderer Infektionskrankheiten $[8,9]$. Dieser Unterschied wurde von Gristina [10] eindrucksvoll am Beispiel der ersten künstlichen Herzen dargestellt, die alle aufgrund von Infektionen versagten. Costerton [4] hat dazu die wesentlichen Erkenntnisse für die bakteriellen Mechanismen beschrieben. Bakterien, die in der Lage sind, Oberflächen von Fremdkörpern zu besiedeln, tun dies, indem sie
Tabelle 8

\begin{tabular}{l} 
Vorteile des einzeitigen \\
Prothesenwechsels bei \\
periprothetischer Infektion \\
\hline Weniger belastend für den Patienten \\
\hline Geringeres Komplikationsrisiko \\
\hline Geringere Kosten \\
\hline Bessere Bedingungen bei der \\
Reimplantation \\
\hline Bessere funktionelle Ergebnisse \\
\hline Gleiche Resultate \\
\hline
\end{tabular}

Tabelle 9

Indikationen für den zwei- oder mehrzeitigen Wechsel

Kein präoperativer Keimnachweis möglich

Resistenz der Keime erlaubt keine topische Antibiotikatherapie

Allergien beim Patienten erlauben nicht die erforderliche Antibiotikatherapie

Extreme Ausbreitung der Infektion ins kleine Becken > reduziertes Débridement

von der planktonischen, frei beweglichen Phase in die sessile übergehen. Dabei bilden sie Biofilm aus und haben damit eine irreversible Besiedelung der Prothese erreicht. Antibiotika und die körpereigene Abwehr sind in diesem Stadium nicht mehr in der Lage, diese Bakterien zu eliminieren. Für die Therapie ergibt sich daraus, dass der Fremdkörper zusammen mit allem infizierten Hart- und Weichgewebe entfernt werden muss, um die Infektion zu beherrschen.

Therapie. Buchholz [1] hat 1969 begonnen, dem Knochenzement Antibiotika beizumischen, mit denen Wirkstoffkonzentrationen vor Ort erzeugt werden, die durch eine systemische Gabe nicht erzielt werden können. Dieses Prinzip wurde zeitgleich zur Prophylaxe und Therapie eingesetzt. Im weiteren Verlauf entwickelte Buchholz daraus das Konzept des einzeitigen Wechsels [2], wobei jetzt unter Antibiotikaschutz direkt reimplantiert wurde.

Grundbedingung für den erfolgreichen einzeitigen Prothesenwechsel bei periprothetischer Infektion ist die präoperative Keimidentifikation. Das Antibiogramm muss Empfindlichkeit auf ein zementgängiges Antibiotikum ausweisen. Der
2. Schritt ist die Entfernung allen Fremdmaterials in Verbindung mit einem radikalen Débridement. Drittens wird reimplantiert unter Verwendung eines speziell antibiotikaverstärkten Zements nach Antibiogramm. Die 4. und letzte Säule des Konzeptes ist die postoperative systemische Antibiose, ebenfalls selbstverständlich individuell nach Antibiogramm.

Der einzeitige Wechsel als ,golden standard" hat anerkannte und nachgewiesene Vorteile, sodass mehrzeitiges Vorgehen Ausnahmesituationen vorbehalten bleiben sollte (• Tabelle 8 ).

Vorteile. Die Vorteile lassen sich subsummieren als geringere Belastung für Patient, Arzt und Kostenträger bei gleichen Ergebnissen (• Tabelle 2).

Die physische und psychische Belastung des Patienten ist auf einen Eingriff begrenzt, und der Patient erfährt das Ergebnis sofort. Die Phase der Unsicherheit, ob und wann reimplantiert werden kann, entfällt. Die Erfolgsbeurteilung einer Operation nicht nur nach Gelingen oder nicht Gelingen, wie bis etwa vor einem Jahrzehnt, sondern auch nach dem Komfort, wie das erreicht wird, ist ein wesentlicher Gesichtspunkt [7].

Vielfach bestehen die Patienten unbeirrbar auf der Versorgung in einem Schritt. Operationsimmanente Risiken wie Thrombose, Nerven- und Gefäßläsionen, Wundheilungsstörungen etc. werden minimiert. Das Ausmaß der Kostenreduktion ist erheblich.

Die sofortige Reimplantation ist für den Chirurgen sehr viel komfortabler als ein Zweiteingriff. Die anatomischen Verhältnisse sind klar und nicht durch Verklebungen, Vernarbungen und Verknöcherungen beeinträchtigt und verschlechtert. Zudem erübrigt sich das Zweitdébridement, wie bei mehrzeitigem Vorgehen erforderlich, und der Substanzverlust im Knochen- und Weichteilbereich wird reduziert. Es resultiert ein besseres funktionelles Ergebnis [12]. Auch die sofortige endgültige physikalische Therapie begünstigt den Verlauf gegenüber einer Phase der Entlastung des Gelenkes bzw. sogar wochenlanger Immobilisation.

Für das mehrzeitige Vorgehen gibt es Indikationen aus medizinischer Sicht, die eingehalten werden sollten (• Tabelle 9 ). 
Antibiotikatherapie. Adjuvante topische und systemische Antibiotikatherapie als Schutz für das neue Implantat und als Vervollständigung des Débridements im Weichteilbereich sind nur effektiv zu verwenden, wenn die Keimsituation bekannt ist und damit ein gezielter Einsatz von Antibiotika möglich ist. Bei unbekannter Keimlage ist man angewiesen auf das radikale Débridement und eine kalkulierte Antibiotikatherapie, die selbstverständlich auch greifen kann. Durch intensive Probengewinnung und -bearbeitung im Rahmen des ersten Eingriffs lässt sich dann oft eine Keimidentifikation erreichen und die Antibiotikatherapie entsprechend adaptieren. Gelingt dies nicht, so kann es trotz klinischer und makroskopischer Unbedenklichkeit immer noch Überraschungen und Misserfolge bei der Reimplantation geben.

Spezialfälle. Außergewöhnliche Resistenzlagen bei Keimen und analog spezielle Antibiotikaallergien beim Patienten schaffen eine höchst komplizierte Situation. Der Ausweg muss immer im Einzelfall unter Führung eines infektiologisch erfahrenen Mikrobiologen gesucht werden. Hier kann es durchaus zu unbefriedigenden Lösungen mit Infektpersistenz oder Gliedmaßenverlust kommen.

Zeigt sich intraoperativ bei der Hüftrevision ein azetabulärer Defekt mit einer lokal weit ins kleine Becken fortgeschrittenen Weichteilinfektion mit begrenzter Möglichkeit für ein radikales Débridement, sollte primär keine neue Pfannenrekonstruktion diesen unsicheren Bereich verschließen. Hier ist eine intraoperative Umstellung auf zweizeitiges Vorgehen zu empfehlen.

\section{Schlussfolgerungen}

Das Prinzip der Therapie der periprothetischen Infektion ist nicht zu reduzieren auf die Frage, ob ein- oder mehrzeitig vorgegangen wird. Entscheidend ist, dass, von Ausnahmen abgesehen, die infizierte Prothese entfernt wird und ein radikales Débridement durchgeführt wird. Diese chirurgische Therapie muss von einer qualifizierten Antibiotikatherapie begleitet werden, um den Erfolg des chirurgischen Vorgehens zu sichern. Voraussetzung ist für beide Verfahren, dass ein erfahrenes Team von Chirurgen, ein qualifiziertes mikrobiologisches Labor und ein klinisch tätiger Mikrobiologe oder Infektiologe die jeweilige Therapie optimieren können.

Das Postulat nach der einzeitigen Versorgung ist vor diesem Hintergrund zu sehen, aber auch das mehrzeitige Vorgehen sollte Kompetenzzentren vorbehalten sein. Ziel muss es sein, allen Patenten, die an einer periprothetischen Infektion erkrankt sind, eine zeitgerechte adäquate Therapie in einem solchen Zentrum zu ermöglichen. Nur so können die Behandlungsergebnisse in der Zukunft stabilisiert und verbessert werden. Es profitiert in erster Linie der Patient, für den wir mitverantwortlich sind, und durch die erhebliche Kostenreduktion die Allgemeinheit.

Bei der gegenwärtigen Vergütung, die davon ausgeht, dass diese Fälle gleichmäßig über alle Krankenhäuser verteilt sind und im Rahmen einer Mischkalkulation für diese Institutionen die Vergütung damit betriebswirtschaftlich hinreichend ist, sind solche Kompetenzzentren nicht finanzierbar, da hier kostenträchtige Fälle ohne finanziellen Ausgleich konzentriert werden. Solange diese Zentren nicht kostendeckend betrieben werden können, ist die volkswirtschaftlich sinnvolle Entwicklung zu Kompetenzzentren weder attraktiv noch wirtschaftlich möglich. Dieser Gedanke muss den Kostenträgern und den politisch Verantwortlichen deutlich gemacht werden.

\section{Korrespondierender Autor Dr. J. Wodtke}

ENDO-Klinik, Holstenstraße 2, 22767 Hamburg E-Mail: Dr.J.Wodtke@t-online.de

\section{Danksagung}

Für die Errechnung der genauen DRG-Erlöse danke ich Dr. Klüber.

Interessenkonflikt: Der korrespondierende Autor versichert, dass keine Verbindungen mit einer Firma, deren Produkt in dem Artikel genannt ist, oder einer Firma, die ein Konkurrenzprodukt vertreibt, bestehen.

\section{Literatur}

1. Buchholz HW, Engelbrecht H (1970) Über die Depotwirkung einiger Antibiotika bei Vermischung mit dem Kunstharz Palacos. Chirurg 41:511-515

2. Buchholz HW, Gartmann H-D (1972) Infektionsprophylaxe und operative Behandlung der schleichenden tiefen Infektion bei der totalen Endoprothese. Chirurg 43: 446-453

3. Charnley J (1979) Low friction arthroplasty of the hip. Springer, Berlin Heidelberg New York

4. Costerton JW, Geesey GG, Cheng K-J (1978) How bacteria stick. Sci Am 238: 86-95

5. Crockarell JR, Hanssen AD, Osmon DR, Morrey BF (1998) Treatment of infection with debridement and retention of the components following hip arthroplasy. J Bone Joint Surg 80-A: 1306-1313

6. Daub D (2003) Das Chefarztsystem im Zeitalter der DRGs. Chirurg BDC 42:M 207-M 211

7. Fraser SC (1993) Quality of life measurement in surgical practice. Br J Surg 80: 163-169

8. Frommelt L (2000) Periprosthetic infection - bacteria and the interface between prosthesis and bone. In: Learmonth ID (ed) Interfaces in total hip arthroplasty. Springer, London, pp 153-161

9. Frommelt L (2004) Prinzipien der Antibiotikabehandlung bei periprothetischen Infektionen. Orthopäde 33: 822-826

10. Gristina AG, Naylor PT, Myrvik QN (1990) Biomaterial-centered infections: microbial adhesion versus tissue integration. In: Wadsström T, Eliassson I, Holder I, Ljungh A (eds) Pathogenesis of wound and biomaterial-associated infections. Springer, London, pp 193-216

11. Hendrich C, Frommelt L (2004) Keim-orientierte Antibiotikatherapie bei Protheseninfektionen. In: Hendrich C, Frommelt L, Eulert J (Hrsg) Septische Knochen- und Gelenkchirurgie. Springer, Berlin Heidelberg New York Tokio

12. Langlais F, Lambotte JC, Thomazeau H (2003) Treatment of infected total hip replacement. In: Lemaire R, Herom F, Scott J, Villar R (eds) European instructional course lectures 6 . The British Society of Bone and Joint Surgery, London, pp 158-167

13. Mohr V (2004) „Leistungen schwanken ganz erheblich": Interview. Spiegel 6/2004: 137

14. Sculco TP (1993) The economic impact of infected total joint arthroplasty. In: Heckman JD (ed) Instructional course lectures 42. American Academy of Orthopaedic Surgeons, Rosemont/ll, pp 349-351

15. Steinbrink K, Mella-Schmidt C (1989) Stellenwert der Spül-Saugdrainage bei der Behandlung des Frühinfekts von Gelenkimplantaten. Chirurg 60: 791-794

16. Zimmerli W, Ochsner PE (2002) Management of infection associated with prosthetic joints. Infection 30: 99-108 